\title{
AN ENSEMBLE CLASSIFICATION APPROACH WITH SELECTIVE UNDER AND OVER SAMPLING OF IMBALANCE INTRUSION DETECTION DATASET
}

\author{
Priyanka Tripathi ${ }^{1}$ and Rajni Ranjan Singh Makwana ${ }^{2}$ \\ ${ }^{1}$ Madhav Institute of Technology and Sclence, CSE \& IT, Gwalior(M.P.), India \\ ${ }^{2}$ Madhav Instıtute of Technology and Sclence, CSE \& IT, Gwalior(M.P.), India \\ ${ }^{1}$ priyankatripathi323@gmail.com, ${ }^{2}$ ranjansingh06@gmail.com
}

\begin{abstract}
KDD CUP 99 dataset is a popular benchmark dataset was introduced at the third international knowledge discovery and data mining tools competition. It widely utilized for the improvement of intrusion detection strategies. The dataset is divided into four type of categories from all attacks which are Probe, DoS, R2L \& U2R. In addition with these attack categories one more category normal is also included in the dataset to represent normal traffic. In the dataset R2L and U2R categories consists of very less tuples in comparison with others. Therefore there is a need for oversampling. Similarly remaining categories should be under sampled to mitigate the class imbalance of the dataset. Synthetic minority oversampling technique (SMOTE) is utilized with different ratios from $50 \%$ to $1000 \%$ for rare classes U2R \& R2L and supplied to the ensemble classifier (Adaboost and random forest). The experiments using machine-learning techniques were conducted using the best ratios. The results using the proposed method were significantly better than those of previous approach and other related work.
\end{abstract}

Keywords - Data mining, Intrusion Detection System (IDS), NIDS(Network based Intrusion Detection System), Weka, Smote, Attacks, Rare Class, Imbalanced Data, KDD Cup 1999

\section{INTRODUCTION}

Detecting network intrusion alludes to the arrangement of the strategies accustomed to separate attacks against PCs \& networks. In this way, IDS distinguishes antagonistic activities in a network. Notwithstanding the attacks identification, this framework have to anticipate their malevolent impacts, otherwise help prevent humans from playing this role into $\mathrm{n} / \mathrm{w}$ administrator. Commonly, even essential systems are perplexing frameworks and the further development of the Internet has made it hard to build an absolute comprehension of the framework.

As of late, the imbalanced learning issue has created a lot of enthusiasm from the scholarly world, industry, and government subsidizing organizations. The primary issues exist \& further discover the classifiers that are able to gain as of imbalanced information devoid of disregarding minor classes. In the direction of manage these issue, numerous arrangements have been given, for example, instance of the testing techniques, objective function strategy, kernal-base strategy \& dynamic learning technique. Sampling strategies still the most generally utilized strategy to manage the issue of imbalanced class. Rather

Received: September 15, 2019

Reviewed: November 20, 2019

Accepted: December 3, 2019

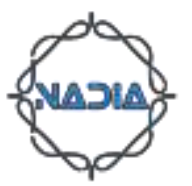


than this issue, one more issue of error misclassification utilized inside learning framework could likewise give contrarily into diminishing precision as well as learning nature.

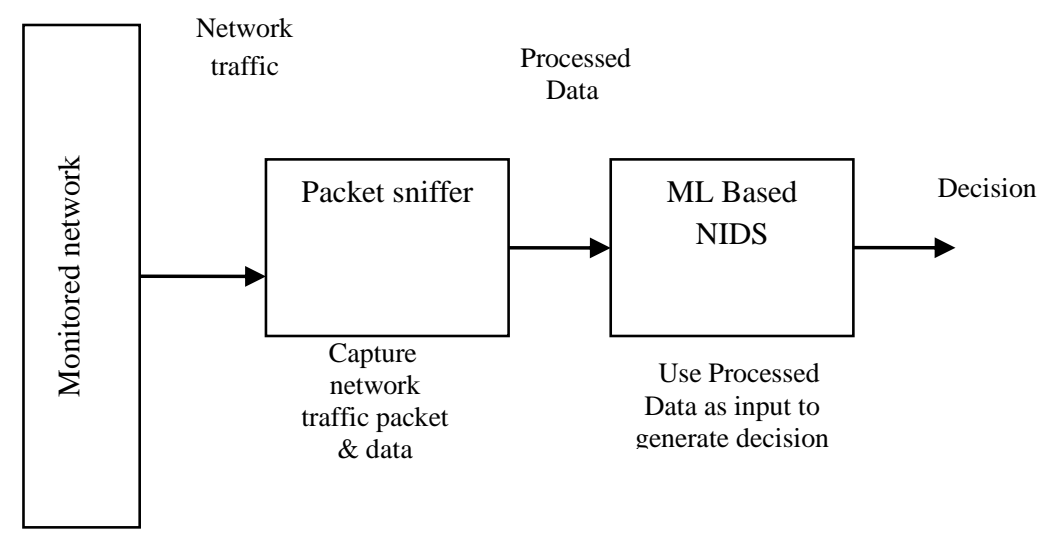

Fig. 1 Machine Leaning Algorithm for NIDS [5]

The remaining articles have been organized in the following form. Section 2 KDD Cup 1999 explains the related functions on the dataset and class imbalance. In Section 3, we recommend using a sample SMOTE ratio to create a numerical model and a new method. In Section 4, we interpret our experimental environment, processes and outcomes. Finally conclude the paper in the Section 5.

AlebachewChiche and Million Meshesha(2017) proposed an intelligent intrusion detection system which can predict attacks in the network and suggest the proper corrective actions for predicted attacks. The system is developed by integrating data mining model and knowledge based system for detecting intrusion types. A model is constructed to predict the intrusion detection is proposed that uses four classifiers MLP, Naive Bayes, Decision tree using J48 and JRip algorithm using rule induction. Dataset used are samples from MIT Lincoln laboratory were collected. Further, the knowledge for prevention techniques are acquired from domain experts and document analysis. The proposed system achieves 91.34 and 85 percent on system performance testing and user acceptance testing respectively. The result is promising to design an intelligent NIDP system by integrating data mining with knowledge based system. Evaluation results show that the proposed system registers $91.43 \%$ accuracy in network intrusion detection and $85 \%$ in user acceptance testing. This indicates proposed system performance is promising for plan intelligent network IDS that can effectively predict and provide a prevention mechanism.

BingHao Yan et al. (2017) to settle data imbalanced attributes in interruption recognition as of information point \& afterward newer district versatile SMOTE calculation has projected to an answer. In the meantime, consecutive backward selecting method was utilized for accelerate recognition procedure by evacuating unnecessary features. Exploratory outcomes demonstrated RA-SMOTE calculation could adequately enhanced rare sample recognition rate, for example, u2l \& r2l usinh NSL-KDD dataset also outflanks additional ID techniques. It has as well revealed RA-SMOT algo receives greatest performance in compare to past algorithm to deal among the unbalanced setback

Yong Sun and Feng Liu (2016) to overcome the problem of noise with bounded data effects proposed a new SMOTE-NCL strategy. It looks at aftereffects of projected strategies within most recent techniques for detecting intrusion. Trial results likewise demonstrate that the new technique has preferred execution over the existing SMOTE strategy in IDS. It can improve the execution of the interruption location, particularly for the identification of minority attacks.

ShilpaBahl and Deepak Dahiya(2016) actualized relationship based feature selection 
utilizing 6 hunt algorithms for developing a successful and exact IDS. By the use of 6 hunt procedures chose only 6 attributes subsets from the specialty space. A foreseen subset has removed as of 6 distinguished subset utilizing rationale for mainly features regular event. Two famous classifier, Random tree \& NB classifier have been applied for comparison. The results have been compared to 6 different subsets, expected subsets, and full 41 features. The planned least subgroup with twelve features showed significant enhancement for total IDS performance to detect $\mathrm{u} 2 \mathrm{r}$. In addition, total time taken to detecting attacks is too low which is beneficial to take an essential action in against these intrusion attacks by administrator.

SireeshaRodda and Uma Shankar Rao Erothi(2016) explores the past works in the use of AI methods for structure proficient NIDS. It additionally gives the review for nsl_kdd data-set. This data-set assessed more than 4 prominent order procedures $\&$ it has seen that highest unbalanced classes haven't appropriately characterized. Henceforth, specific strategies for effectively classifying uncommon classes are necessary.

T. Herawan et al. (2014) use the true NIDS dataset, KDD Cup 1999, to assess proposed IDS with regards to data mining. In any case, the imbalanced class conveyance of the informational index prompts an uncommon class issue. The issue causes low recognition (grouping) rate to uncommon class, especially r2l \& u2r. Two ordinarily utilized inspecting strategies to moderate the uncommon class issue were assessed in this exploration, to be specific, (1) under-sampling \& (2) over-sampling. In any case, these two strategies were less powerful in alleviating the issue. The reasons for such execution are exhibited in this paper.

L'idio et al. (2012) mimic an analyzer to the system traffics which one also IDS part. This setting talks about and exhibits as a commitment, not just the classifiers that were utilized in the issue of interruption identification, yet additionally the underlying phase of preparing the data. Hence, they tried the execution of three classifiers over kddcup99 standard ID dataset also chose classifiers which is top. They at first tried a decision tree \& NN utilizing by the dataset, recommending enhancements via decreasing the quantity to ascribe in range of 42-27 thinking about just two classes of discovery, normal \& abnormal. At last, they tried the decision tree \& Bayesian network classifiers thinking about 5 assault classes: normal, dos, u2r, r21 \& probe. Exploratory outcomes demonstrated that calculations utilized accomplished highest detection rates with a huge decrease of FP to various sorts of the $\mathrm{n} / \mathrm{w}$ intrusions utilizing constrained computing assets.

\section{PROPOSED METHODOLOGY}

\subsection{FEATURE SELECTION}

Basically there are two methodologies in choosing best feature subset, i.e. filter, and wrapper approach. The Filter approach is used to generate the optimized set of feature by filtering the features prior to the machine learning procedure. Then again, the wrapper approach looks for a streamlined list of capabilities using a learning algorithm. Set of features using learning algorithm Specifically Consistency Subset Evaluator (CSE) and Correlation Based Feature Selection Subset Evaluator (CFSE), consists of two essential sub-evaluation evaluators. CSE estimates the discrepancy for given various class labels to the feature set. Though, CFSE estimates prescient capacity of all features, alongside the degree of redundancy among them.

\subsection{Classification}

This includes trained datasets learning which labels as class from data. A classifier has made of later learning \& this is used for classifying unlisted data inside the test dataset. AdaBoost and Random Forest Classifiers can be built for categorization reason. 
Accurateness of the classification has achieved to calculate the execution classifier execution.

AdaBoost. In AdaBoost, the aim is to combine dierent weak learners orclassifiers (hi(x)) together to improve the classification performance, where hi(x)is a single classifier. Each weak learner is trained using a simple set of training samples.Each sample has a weight and the weights of all samples areadjusted iteratively. AdaBoost iteratively trains weak learners and calculate a weight foreach one, and this weight represents the robustness of the weak learner.

The AdaBoost algorithm has the following main steps:

Sampling step: In this step, some samples (Dt) are selected from the training set, where Dt is the set of samples in the iteration $t$.

Training step : In this step different classifiers are trained using Dt \& the error rates ( $\epsilon \mathrm{i})$ for each classifier are calculated.

Combination step: Here all trained models are combined.

Random Forest: Depending on estimates of Random Forest (RF) tree is a combination of Predictors such that each tree vector test such a way that a mixture of tree Indicators each tree an arbitrary and equally spread to all the trees. Simplification error for forests becomes potentially widespread for tree volume in the forest as much as possible. This simplification error for tree classifiers forest depends on quality of single-tree inside forest \& relationship between's them. The error rate is adjusted using a random selection of features to split each node, which is AdaBoost friendly, but stronger in relation to noise.

Officially, RF is a predictor that includes set of random base regression trees. $\{\mathrm{rn}(\mathrm{x}, \Phi \mathrm{m}, \mathrm{Dn}), \mathrm{m} \geq 1\}$, here $\Phi 1, \Phi 2, \ldots$ defined as randomized factor $(\Phi)$ outcomes. Aggregated regression estimation is calculated by joining of the random trees r $\mathrm{n}(\mathrm{X}, \mathrm{Dn})=\mathrm{E} \Phi[\mathrm{rn}(\mathrm{X}, \Phi, \mathrm{Dn})]$,

Where, EФ representing the expectation for random factor, conditionally probable on the $\mathrm{X} \&$ dataset Dn.

\section{Proposed Algorithm}

Step:1 Input original training dataset.

Step:2 Separate Each class (dos,normal,probe,u2r,r2l) data

Step:3 This removes $\mathrm{r} 21$ class, same way remove 4 classes and save one. Repeat this till all the classes are separated.

Step:4 Remove duplicate from DOS and Normal class.

Step:5 Then merge all data of class files.

Step:6 Open Merged File

Step:7 Select specific attributes as specified in paper and remove extra attributes.

Step:8 Discretized the data

Step:9 Apply Smote for 50\% for rare classes R2L U2R.

Step:10 Repeat step 9.

Step:11 Apply cost sensitive classifier and select AdaBoost with Random forest.

Step:12 Then Classify using AdaBoost with Random forest for SMOTE on various \% of rare classes.

Step:13 Classified instances.

Step:14 Results.

\section{RESULT ANALYSIS}


In the result analysis, the experiment of proposed work performed by using WEKA.

There KDDCup 1999 dataset was used for experiment. This dataset have divided into 4 type of categories from all attacks which are Probe, DoS, R2L, \& U2R. The normal traffic of $\mathrm{n} / \mathrm{w}$ inside dataset termed by normal.

Table I. KDD Cup dataset distributed classes over $10 \%$ of overall dataset

\begin{tabular}{ccc}
\hline CLASSES_NAME & TRAINED_SET (TRNS) & TESTED_SET (TEST) \\
\hline Normal & 97,277 & 60,593 \\
Probe & 4,107 & 4,166 \\
u2r & 52 & 228 \\
Dos & 391,458 & 229,853 \\
r21 & 1,126 & 16,189 \\
Sum & 494,020 & 311,029 \\
\hline
\end{tabular}

Most of the research in IDS research including in this work, favored lower edition to actual dataset (ten percentage of the initiative dataset) provided by the competition organizer. Dataset in reduced form for distributed classes has been displayed in Table 1. Class distribution for a lot of imbalanced displayed in training set (denoted as TRNS). The tested_set (i.e. TEST) don't track distribution possibility for trained_set \& further attack has be supplemental for various class to build an IDS costing most practical.

Table II. Comparing the initative trained_set (TRNS) with the KDDCup99 after UnderSampling Trained_set (TRNS_US)

\begin{tabular}{ccc}
\hline CLASSES_NAME & TRAINED_SET (TRNS) & $\begin{array}{c}\text { UNDER-SAMPLING } \\
\text { TRAINED_SET } \\
\text { (TRNS_US) }\end{array}$ \\
\hline Normal & 97,277 & 87,831 \\
Probe & 4,107 & 4107 \\
u2r & 52 & 78 \\
Dos & 391,458 & 54,572 \\
r21 & 1,126 & 1689 \\
Sum & 494,020 & 148,277 \\
\hline
\end{tabular}

These normal \& dos predominant class have Under-Sampling for adjusting class circulation to TRNS via expelling indistinguishable entries to prevailing class \& brought about a further preparation-set, TRNS_US appeared in table2. An incredible decrease was accomplished for the normal $\&$ dos class, individually. In view of the decrease, the span for TRNS has significantly diminished \& just 148,277 entries ( $30.01 \%$ for TRNS) stayed inside TRNS_US.

Table III. Explanation of concerned attributes from attribute set 


\begin{tabular}{|c|c|}
\hline Attributes & Explanation \\
\hline Period & time taken to connection (sec.) \\
\hline Services & $\begin{array}{l}\text { At receiver end defined } \mathrm{n} / \mathrm{w} \\
\text { service types }\end{array}$ \\
\hline root_shel & $\begin{array}{l}\text { Achieved root shell otherwise } \\
\text { else }\end{array}$ \\
\hline FLAG & $\begin{array}{l}\text { connection situation (it is } \\
\text { normal or there is any error) }\end{array}$ \\
\hline SRC_BYTES & $\begin{array}{l}\text { Tot up data bytes which are } \\
\text { sends from sender to the } \\
\text { receiver }\end{array}$ \\
\hline numb_files_creation & entire creating file operation \\
\hline LOGGED_IN & $\begin{array}{l}\text { successfully login or any } \\
\text { others }\end{array}$ \\
\hline NUM_FAILED_LOGINS & $\begin{array}{l}\text { Entire attempt to login in } \\
\text { failure }\end{array}$ \\
\hline dest_host_reror_rates & $\begin{array}{l}\text { Connection rates including } \\
\text { "REJ" errors }\end{array}$ \\
\hline dest_host_dif_srs_rates & $\begin{array}{l}\text { For different types services for } \\
\text { connections rate }\end{array}$ \\
\hline
\end{tabular}

SMOTE is created for the unbalanced data sets, which is an over-sampling technique in TRNS_US. This includes creating synthetic rare examples of the rarely chosen $\mathrm{k}$ rare neighbors. This research has been used for five by default k. r2l \& u2r have enlarged by fifty -thousands of percentage TRNS_US

Table IV. Obtained rates of detection (in \%) by Ensemble classifiers (Adaboost and Random Forest), wherever r21 \& u2r for TRNS_US be oversample through the smote

\begin{tabular}{|c|c|c|c|c|c|}
\hline Normal & Probe & $\mathrm{U} 2 \mathrm{r}$ & Dos & R2I & $\overline{\text { Observations }}$ \\
\hline 10.0 & 98.2 & 87.2 & 10.0 & 98.6 & $\begin{array}{l}\text { Rare classes } \\
+50 \%\end{array}$ \\
\hline 99.1 & 98.3 & 91.3 & 10.0 & 99.1 & $\begin{array}{l}\text { Rare classes } \\
\quad+100 \%\end{array}$ \\
\hline 99.2 & 98.6 & 93.1 & 10.0 & 99.2 & $\begin{array}{c}\text { Rare classes } \\
+150 \%\end{array}$ \\
\hline 10.0 & 98.1 & 94.9 & 10.0 & 99.4 & $\begin{array}{c}\text { Rare classes } \\
+200 \%\end{array}$ \\
\hline 10.0 & 98.5 & 96.9 & 10.0 & 99.6 & $\begin{array}{l}\text { Rare classes } \\
\quad+400 \%\end{array}$ \\
\hline 99.9 & 98.3 & 97.8 & 10.0 & 99.7 & $\begin{array}{l}\text { Rare classes } \\
\quad+600 \%\end{array}$ \\
\hline 99.9 & 98.3 & 98.3 & 10.0 & 99.8 & $\begin{array}{l}\text { Rare classes } \\
\quad+800 \%\end{array}$ \\
\hline 99.9 & 98.4 & 98.6 & 10.0 & 99.9 & $\begin{array}{c}\text { Rare classes } \\
+1000 \%\end{array}$ \\
\hline
\end{tabular}

In above table it is observed that the detection rate at $1000 \%$ Normal is $99.9 \%$, Probe is $98.2 \%$, Dos is $100 \%$, u2r is $98.6 \%$ and $\mathrm{r} 21$ is $99.9 \%$ which is much higher than the previous one.

Table V. Confusion Matrix 


\begin{tabular}{cccccc}
\hline a & b & C & D & E & Classified as \\
\hline 54564 & 2 & 0 & 6 & 0 & a=DoS \\
48 & 4034 & 1 & 24 & 0 & b=Probe \\
0 & 0 & 68 & 10 & 0 & c=U2R \\
20 & 4 & 5 & 87791 & 11 & d=Normal \\
1 & 0 & 3 & 20 & 1665 & e=R2L \\
\hline
\end{tabular}

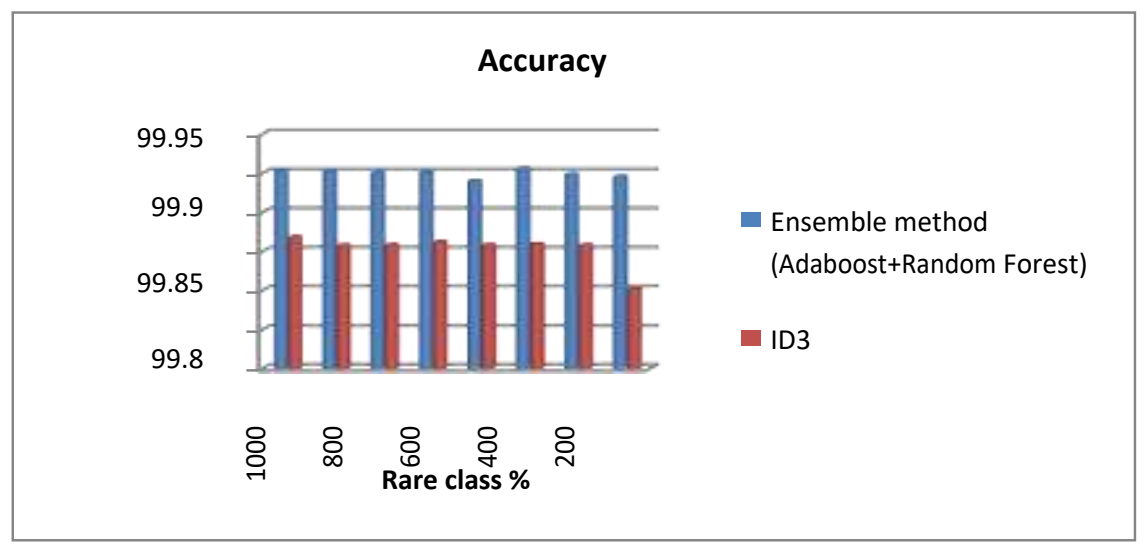

Fig. 2 Comparison of Accuracy between existing and proposed method at various \% of rare class

In previous research where ID3(Khor, K.-C., Ting, C.-Y., \& Phon-Amnuaisuk, S.11) is used, accuracy at $200 \%$ is 99.85 while in ensemble(Adaboost and Random Forest) method accuracy is $99.9 \%$ whereas in ID3 accuracy at $1000 \%$ is $99.89 \%$ while in ensemble method accuracy is $99.9 \%$.

Table VI. Detailed Accuracy by Class

\begin{tabular}{lllllllll}
\hline $\begin{array}{l}\text { TP } \\
\text { Rate }\end{array}$ & FP & Precision Recall & F- & MCC & R O C PRC & Class \\
Re ea & Mrea & \\
& & & & & & & & \\
\hline 1.0 & 0.0 & 0.999 & 1.0 & 0.99 & 0.9 & 1.0 & 0.9 & DoS \\
00 & 01 & & 00 & 9 & 99 & 00 & 99 & \\
0.9 & 0.0 & 0.998 & 0.9 & 0.99 & 0.9 & 0.9 & 0.9 & Probe \\
84 & 00 & & 84 & 1 & 91 & 96 & 91 & \\
0.9 & 0.0 & 0.984 & 0.9 & 0.98 & 0.9 & 0.9 & 0.9 & U2R \\
86 & 00 & & 86 & 5 & 85 & 94 & 81 & \\
0.9 & 0.0 & 0.999 & 0.9 & 0.99 & 0.9 & 1.0 & 1.0 & Normal \\
99 & 01 & & 99 & 9 & 99 & 00 & 00 & \\
0.9 & 0.0 & 0.999 & 0.9 & 0.99 & 0.9 & 1.0 & 1.0 & R2L \\
99 & 00 & & 99 & 9 & 98 & 00 & 00 & \\
0.9 & 0.0 & 0.999 & 0.9 & 0.99 & 0.9 & 1.0 & 0.9 & Weighted \\
99 & 01 & & 99 & 9 & 98 & 00 & 99 & Avg. \\
\hline
\end{tabular}

True Positive rate (TP) of Dos is $100 \%$, Probe is $98.4 \%$, U2R is $98.6 \%$, Normal is $99.9 \%$ and $\mathrm{R} 2 \mathrm{~L}$ is $99.9 \%$.

\section{CONCLUSIOON}

In this work over and under sampling of minority and majority classes are carried out to mitigate the class imbalance problem present in KDD CUP 99 dataset. To boost the performance and best used with weak learners problem; a novel ensemble classifier is proposed. This is the combination of AdaBoost and Random forest classifiers. A comparative analysis if carried out with existing work and it is observed that proposed 
method give better result. In future, work can be extended by introducing Cluster basedSMOTE as a technique for imbalanced class learning above SMOTE within the scope of these datasets. Work can be tested by using various combinations of hybrid classifiers, in future.

\section{REFERENCES}

[1] Cieslak, D. A, Chawla N. V , Striegel, A.: Combating Imbalance in Network Intrusion Datasets IEEE, 2006, pp. 1-6.

[2] H. Haibo , A. Garcia, E.: "Learning from Imbalanced Data", IEEE Transactions On Knowledge And Data Engineering, Vol.2, No.9, September (2009).

[3] C, Chen, Liaw, A. , L. Breiman : Using Random Forest to Learn Imbalanced Data.

[4] I. Chairi , S. Alaoui, A. Lyhyaoui: "Intrusion Detection based Sample Selection for imbalanced data distribution" Second International Conference on the Innovative Computing Technology ,pp. 259-264, (INTECH 2012).

[5] Li J., Qu Y., Chao F., Shum H.P.H., Ho E.S.L., Yang L : Machine Learning Algorithms for Network Intrusion Detection. In: Sikos L. (eds) AI in Cybersecurity. Intelligent Systems Reference Library, vol 151. Springer, Cham,(2019).

[6] Alebachew Chiche, Million Meshesha :"An Intelligent Network Intrusion Detcetion System using Data Mining and Knowledge Based System", Journal of Theoretical and Applied Information Technology, Vol. 95, No. 17 ( 2017).

[7] Yan, B., Han, G., Sun, M., Ye, S. : “A novel region adaptive SMOTE algorithm for intrusion detection on imbalanced problem",3rd IEEE International Conference on Computer and Communications (ICCC), pp. 1281-1286, (2017).

[8] Yong Sun, Feng Liu , SMOTE-NCL: "A re-sampling method with filter for network intrusion detection",2nd IEEE International Conference on Computer and Communications (ICCC), pp. 11571161, (2016).

[9] Bahl S, Dahiya D: "Enhanced Intrusion Detection System for Detecting Rare Class Attacks using Correlation based Dimensionality Reduction Technique", Indian Journal of Science and Technology, Vol 9 (11), pp. 1-10, March 2016,.

[10] Rodda, S., \& Erothi, U. S. R. , "Class imbalance problem in the Network Intrusion Detection Systems". 2016 International Conference on Electrical, Electronics, and Optimization Techniques (ICEEOT), pp. 2685-2687, (2016).

[11] Khor, K.-C., Ting, C.-Y., \& Phon-Amnuaisuk, S. , "The Effectiveness of Sampling Methods for the Imbalanced Network Intrusion Detection Data Set. Recent Advances on Soft Computing and Data Mining, 613-622, (2014).

[12] de Campos L.M.L., de Oliveira R.C.L., Roisenberg M. : "Network Intrusion Detection System Using Data Mining” In: Jayne C., Yue S., Iliadis L. (eds) Engineering Applications of Neural Networks. EANN 2012. Communications in Computer and Information Science, vol 311. Springer, Berlin, Heidelberg, pp. 104- ((2012).

[13] Kok-Chin Khor, Choo-Yee Ting, Somnuk-Phon Amnuaisuk : "Forming an Optimal Feature Set for Classifying Network Intrusions Involving Multiple Feature Selection Methods”, IEEE, pp. 179-183, (2010).

[14] Alaa Tharwat :"AdaBoost: an Overveiw",researchgate, pp. 1-72,(2018).

[15] Leo Breiman: "RANDOM FORESTS", Statistics Department University of California Berkeley, CA 94720, pp. 1-33, (2001).

[16] Biau G. : “Analysis of a Random Forests Model”, Journal of Machine Learning Research, no. 13, pp. 1063-1095, (2012). 